\title{
Applying a Dynamic Model of Situated Cognition to the Investigation of Mishaps
}

\author{
Nita Lewis Miller \\ Naval Postgraduate School \\ Monterey, CA 93943 \\ Lawrence G. Shattuck \\ United States Military Academy \\ West Point, NY 10996
}

\begin{abstract}
Complex systems will, inevitably, experience failures. The cause of these failures or mishaps may be labeled 'operator error,' but often they are actually caused by the confluence of technological, situational, individual, and organizational factors. Several models and theories of human error have been proposed over the years and are reviewed in this paper. The authors propose another model, the Dynamic Model of Situated Cognition (DMSC), to explain how complex systems fail. Miller and Shattuck (2004) developed the DMSC in an effort to link technological aspects of a system to the perceptual and cognitive aspects of that system. They illustrated the model by applying it to the USS Stark incident and to a military command and control simulation (Shattuck and Miller, 2004). The model also appears to have utility as a retrospective explanatory tool to identify when and where things went wrong. In this paper, the authors describe the DMSC as it relates to the analysis of error in complex systems and apply it to the February 2001 mishap in which the U.S. Navy submarine USS Greeneville collided with the Japanese motor vessel Ehime Maru off the coast of Oahu, Hawaii.
\end{abstract}

\section{INTRODUCTION}

Complex cognitive systems will, inevitably, experience failures. The proximate cause of these failures or mishaps are often labeled 'operator error,' but more often than not they are caused by the confluence of technological, situational, individual, and organizational factors. Reason (1990; 1997) argues that complex systems have a series of defenses which are designed to prevent accidents from occurring. Holes in these defenses are created by latent or active failures which set the conditions for a trajectory that can lead to mishaps. Other researchers have examined error in complex systems and have developed their own models of how and why accidents occur (Senders \& Moray, 1991; Woods, Johannesen, Cook, \& Sarter, 1994; Doerner, 1996; Snook, 2000; Strauch, 2002; Dekker, 2002).

Miller and Shattuck (2004) developed a Dynamic Model of Situated Cognition linking the technological aspects of a complex system to the perceptual and cognitive aspects of the system. They illustrated the model by applying it to a military command and control simulation. Data and information flowed (and sometimes were prevented from flowing) through the model resulting in decision makers' understanding of the situation. This model is not only useful for examining the flow of data and information as events unfold in a complex system, it also appears to have utility as a retrospective explanatory mechanism to identify when and where things go wrong. This paper reviews approaches to explaining human error, presents further details on the Dynamic Model of Situated Cognition, and demonstrates how the model can be used to retrospectively account for mishaps.

Senders and Moray (1991) suggest that hardware failures can contribute either directly or indirectly to abnormal system states and that human error can be proximate or remote. Their causal network for error analysis includes both technological (hardware) and human components but it is not clear how those components interact with one another as an event unfolds. The authors propose that investigators construct a causal chain which begins at the point of failure and proceeds backward in time to events that might have contributed to the mishap.

Strauch (2002) reviews several of the leading theories of error in complex systems. He states that "operator errors are the logical consequences of antecedents or precursors that had been present in the systems at the time they were committed" (p. 16). The emphasis on system antecedents is a relatively recent phenomenon in the field of error investigation. Antecedents hidden in the system are detected when they influence an operator's performance. Investigators have also expanded the scope of their analysis by broadening both what they consider to be the system and what they deem to be the relevant window of time. Strauch lists six elements that comprise a system (after Moray, 1994, 2000), each more removed from the actual failure event than the preceding element: equipment; individual operator; multi-operator team; company and management; regulator; and, social and cultural factors.

Woods, et al. (1994) characterize a complex system as having a sharp end, where operators interact with technology, and a blunt end, which includes managers, designers, and administrators who are spatially and temporally distant from the operators. They list fourteen premises of human error. Included in their list are the following:

- Erroneous actions and assessments are the start of the investigation, not the end.

- Erroneous actions are a symptom, not a cause.

- There is a loose coupling between process and outcome.

- Incidents evolve through the conjunction of several failures/factors.

- Some of the contributing factors to incidents are latent in the system. 
- Error tolerance, error detection, and error recovery are as important as error detection.

- The design of artifacts affects the potential for erroneous actions and paths toward disaster.

Reason $(1990 ; 1997)$ describes error in terms of a failure in a system's defenses. He postulates that a system may have multiple defenses, each of which may have holes in them due to either latent or active failures. If the holes line up, it is possible for an error trajectory to pass through the system, which will result in an accident or mishap, hence the analogy of a 'Swiss cheese' model. Reason describes a system as having three levels (the person, the workplace, and the organization). Causality flows from the organization to the person; accident investigations typically flow from the person back to the organization.

Recent work by Wiegmann and Shappell (2003), based on Reason's theoretical framework, focuses on a methodology for conducting aviation accident investigations. The Human Factors Analysis and Classification System (HFACS) expands on Reason's work by offering a taxonomic approach to classifying the nature of the holes in his 'Swiss cheese' model. HFACS has received considerable attention in the military and civil aviation communities and has been recently adopted as an accident investigation tool by several of these US and international communities.

In general, the human error models described above consider systems to have distal and proximate elements. These models may also include both human and technological components. Flaws in these models may lie dormant or go unnoticed until certain activities or precipitating events uncover them. These models do not necessarily describe systems in a dynamic manner and they do not address how systems are designed to perform. In Figure 1, the Dynamic Model of Situated Cognition (Miller and Shattuck, 2004) describes total human system performance, incorporating the notion that this process is dynamic, with feed-forward and feedback loops operating continuously. The authors have previously used the DMSC to illustrate design flaws and the flow of information in a system. In this paper, the authors argue that the model can also be used to explain how mishaps occur.

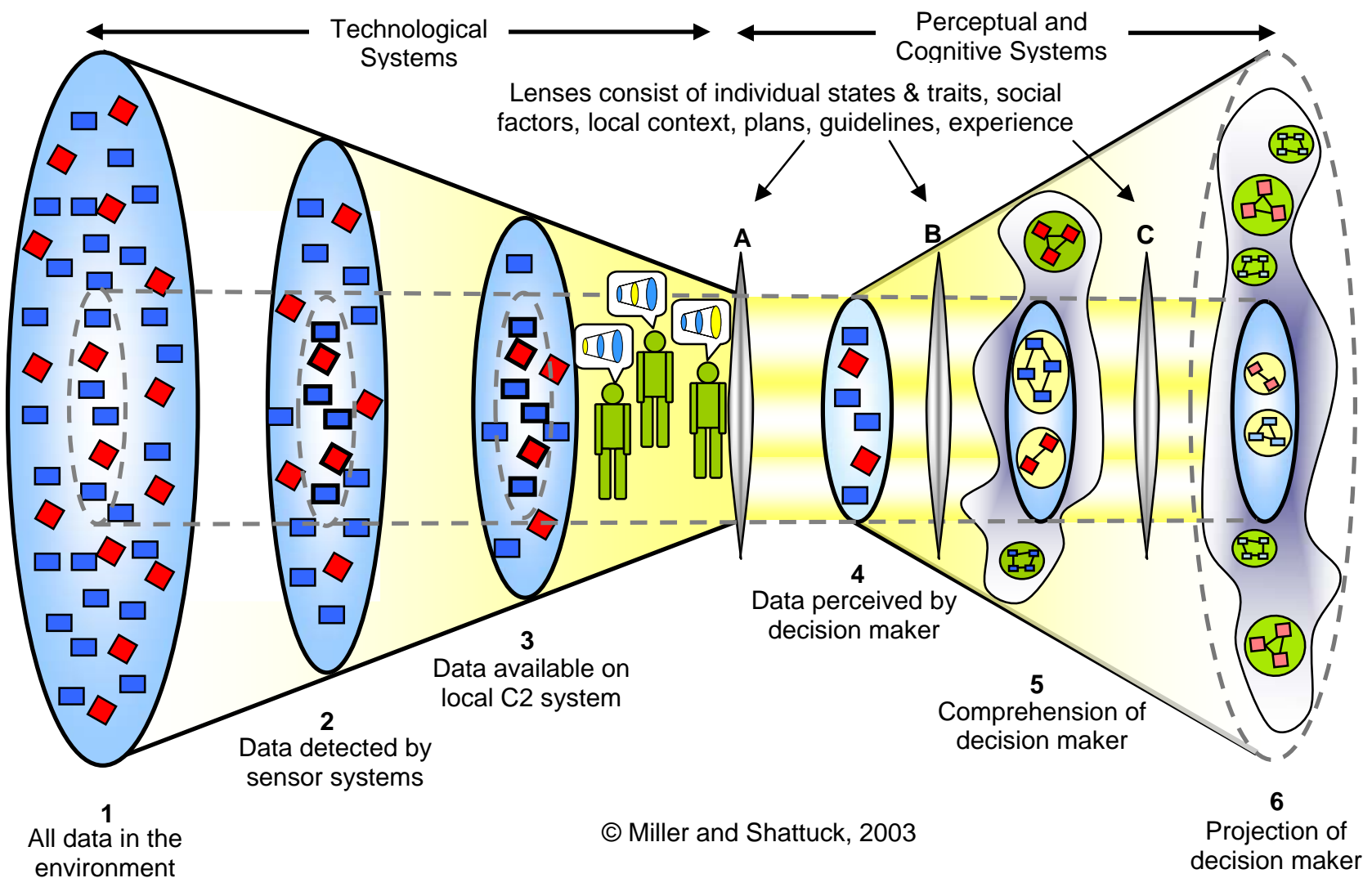

Figure 1. A Dynamic Model of Situated Cognition.

\section{TECHNOLOGICAL PORTION OF THE SYSTEM}

The DMSC is applicable to virtually any domain in which technology and humans combine to accomplish a stated goal. The authors, however, have chosen to explain and illustrate the model in a military command and control (C2) setting. The large oval on the left side of the figure (Oval 1) depicts ground truth, i.e., everything in the world. Ground truth is dynamic in nature, changing with spatial and temporal progression although it may be captured or frozen at points in time. There is no error or uncertainty with respect to Oval 1. Simulations allow for the accurate representation of ground truth. In the real world, however, it is understood that Oval 1 cannot be measured with complete precision and accuracy. 
The blue rectangles and red diamonds in Oval 1 represent individual data elements for friendly and enemy entities. While are other things are present in the world (e.g., noncombatants, weather, terrain, friendly and enemy intent), they have been excluded from the figure for simplification.

The next oval (Oval 2) shows the elements detected by the technological systems. Note that this oval is depicted as a subset of the first oval. However, it is not apparent what portions of ground truth are being detected and what items remain undetected. Error or uncertainty can first enter the model at Oval 2, taking various forms. Oval 2 is a subset of Oval 1 for a variety of reasons.

- Not enough sensors available to cover environment.

- Sensors unable to detect due to lack of sensitivity or specificity.

- Enemy activity designed to deceive (spoofing).

- Sensors may malfunction.

As in subsequent sections of the model, inaccuracy or uncertainty in Oval 2 can result in error which may be propagated throughout the rest of the model.

Oval 3 depicts what is displayed on the decision maker's workstation. Again, these data are a subset of what is represented in Oval 2. Users are able to tailor their displays to suit their individual preferences. If the user has the display settings adjusted on the $\mathrm{C} 2$ screens such that information is not displayed, it is possible that important, perhaps vital, information may be hidden or concealed. It may not be obvious to the user which data have been excluded. Oval 3 represents yet another entry point for error or uncertainty into the model.

\section{HUMAN PORTION OF THE SYSTEM}

Three distinct lenses are depicted in Figure 1. Each individual enters a situation with a unique set of lenses. In a military context, the lenses may be thought of as shaped by the following categories: individual traits or characteristics, states or performance characteristics (system, team and individual), experience (societal, cultural and individual), and doctrine and rules of engagement (macro level - the context in the months preceding the incident; micro level - the context in the days and hours preceding the incident). Together, these classes of information influence what is perceived by the user or decision maker and they represent potentially significant sources of error.

Oval 4 represents all the data actually perceived by the decision maker. Perceived data in Oval 4 are a subset of the data available in the environment, the sensor array, the configuration of the local C2 display, and are based on the characteristics of the lens. In addition, information in Oval 4 can be received from other humans, represented by the three figures between Oval 3 and Lens A. These humans can contribute their perceptions, comprehensions or projections.

Perceived data are of little value to the decision maker until they are processed. The same lens components that directed attention and led to perception also influence comprehension. The friendly and enemy icons in Oval 5 have been linked and reorganized, suggesting that they have been processed, representing the comprehension of the data elements that were perceived. The oval is embedded in an amorphous shape, suggesting that there are other possible ways the data could be linked and reorganized that would lead to alternative mental representations of the data.

The final oval (Oval 6) represents the projection into the future or the prediction of the user. Alternate views of the battlefield within the amorphous shape but outside of Oval 5 do not contribute to or influence the prediction of the decision maker. Note that the amorphous shape that surrounds Oval 6 is larger than that which surrounds Oval 5 and contains even more alternatives. This representation depicts the idea that there is much greater uncertainty associated with prediction.

Thus far, the DMSC has been described as a relatively linear process starting in Oval 1 and progressing through Oval 6 but that is far from accurate. There are feedback loops to previous Ovals and Lenses in the DMSC that are intrinsic to its dynamic nature. To illustrate how feedback loops may influence the information flow process, a projection (Oval 6) could lead the decision maker to alter speed or trajectory (thereby changing Oval 1), modify the sensor coverage (Oval 2) or a local C2 display (Oval 3), influence perception of data (Oval 4), or influence comprehension of data (Oval 5). Moreover, both projection (Oval 6) and comprehension (Oval 5) can shape the contents and contours of the decision maker's lenses (A, B, and C). Error may be inected into the system through any of these feedback loops.

\section{APPLICATION OF THE MODEL}

To demonstrate the utility of the DMSC, we examine an actual submarine command and control mishap on the USS Greeneville, using a process tracing methodology to pinpoint where and when the errors occurred, leading to the mishap. This analysis offers an example of how the DMSC can be used to determine how errors enter a system and are then propagated through it.

On February 9, 2001, the U.S. Navy submarine USS Greeneville collided with the Japanese motor vessel Ehime Maru off the coast of Oahu, Hawaii. (See Figure 2 for an organizational chart of the personnel directly involved in the C2 process and in this incident.) The submarine was demonstrating an emergency surfacing maneuver for civilian guests onboard for a seven-hour distinguished visitor cruise. As it rose to the surface, the submarine struck the fishing vessel's aft port quarter, causing the ship to sink in less than 10 minutes. Of the 35 Japanese crew, instructors, and students onboard the Ehime Maru, 26 were rescued while nine remain unaccounted for, presumed dead (Executive Summary of USS Greeneville collision, retrieved 04/14/2005 from http://www.cpf.navy.mil/greeneville.html).

For the purposes of the DMSC application, the following sources of error, while far from exhaustive, will be used:

- Submarine is behind schedule.

- Equipment failure.

- CO is overconfident; influences lenses of crew.

- FTOW manually overrides and enters incorrect surface contact solution data. 


\section{Submarine Behind Schedule}

The problems started when the submarine fell behind schedule by 30 minutes and had less than an hour to get to a pre-designated location. At this point, the commanding officer (CO) of the USS Greeneville made a series of decisions and issued orders that created an artificial sense of urgency in crewmembers in the control room. This elevated time pressure affected the individual lenses (Lens A, B and C on the right side of the model) of the crewmembers, adversely impacting their ability to accurately process the information residing in Oval 4 though Oval 6 (perception, comprehension, and projection). Error enters the model through this process.
One of the decisions made by the $\mathrm{CO}$ was to prepare to come to periscope depth. Mandatory procedures for a submarine to come to periscope depth (PD) require that the Officer of the Deck (OOD) hold a periscope briefing with watchstanders, conduct two good target motion analysis (TMA) legs of about three minutes on each surface contact, provide the necessary report and obtain the CO's permission to proceed to $\mathrm{PD}$. These procedures were known to all crewmen and were part of their lenses. The CO, however, abbreviated these procedures used by the crew to maintain their SA during the PD maneuver.

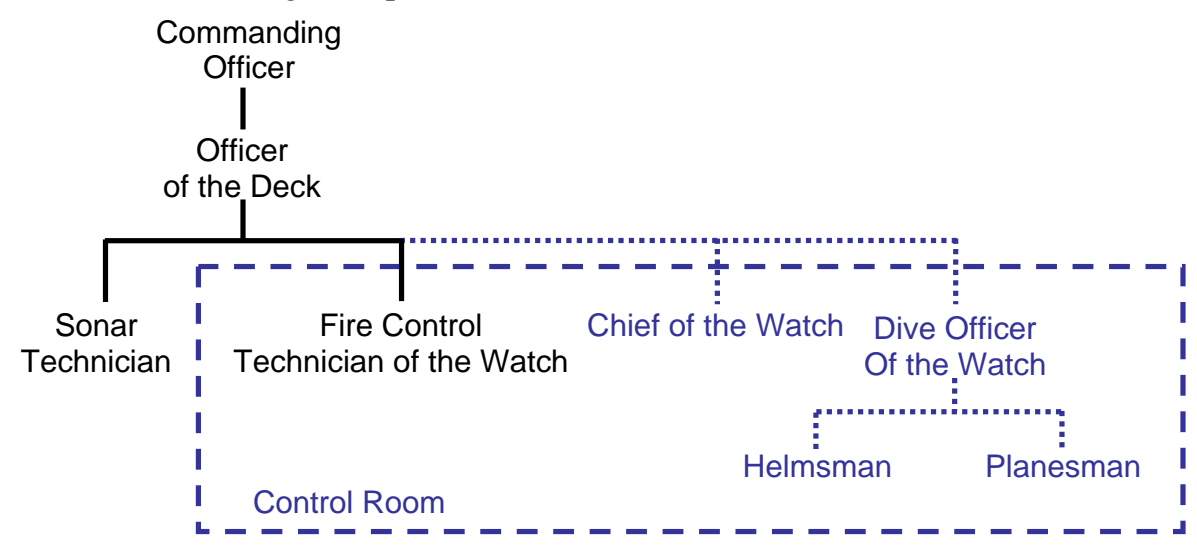

Figure 2. Partial organizational chart for the USS Greeneville.

The CO's decision not only compromised the procedures, it generated feedback loops to other portions of the system which virtually assured the data in the environment (Oval 1) which were detected by the sensors (Oval 2) were inaccurate or incomplete. Hence, the data displayed on the C2 workstations (Oval 3) were also inconsistent with Oval 1; and the perception, comprehension, and projection (Ovals 4, 5, and 6 ) of the crewmembers with regard to the surface contacts were formulated based on erroneous data.

\section{Equipment Failure}

The information on the closing rate of the Ehime Maru was not in Oval 3 (data available on the C2 system) because a critical display system was not working. The Analog Video Signal Display Unit (AVSDU), located in the control room of the submarine where it provides a remote display of sonar data used for surface contact analysis, was inoperative. Since information on the proximity of the Ehime Maru was not available in Oval 3 from the AVSDU, it could not be propagated throughout the rest of the model. Hence, errors may arise either because erroneous data propagate through a system or because data fail to propagate through a system.

\section{Overconfidence of $\mathrm{CO}$}

There were three surface ships in the vicinity of the submarine. Data on two of the surface contacts, including the Ehime Maru, resided in both Oval 1 (ground truth or data in the environment) and in Oval 2 (data detected by sensors) of the DMSC. The third contact was only in Oval 1. The Ehime Maru was closing on the USS Greeneville but at this point this information only was available in Oval 1 and Oval 2. Prior to the CO's decision to surface, the sonar technician reported a new contact to the control team. The third surface contact in Oval 1 had just been detected by the sensors (Oval 2) and was now displayed on the sonar technician's workstation (Oval 3). Although the sonar technician had announced the new contact, this information either did not pass through Lens A in the model or was skewed as it passed through. The result was that neither the CO nor the OOD perceived (Oval 4) the situation properly; they did not recognize that the sonar report was information on a new contact.

The CO then announced to Control that he had a good feel for the contact picture and ordered the OOD to proceed to PD on the same course. The OOD was not given enough time to develop an accurate picture of the surface contact situation. He did not conduct the required periscope brief with watchstanders, missing a valuable opportunity to receive and critically assess important contact information from the sonar tech. He was deprived of input from both the technological agents and the human agents in the system.

Additionally, other crewmembers were not given enough time to do their jobs properly. Upon hearing the sonar technician's report of the new contact, the FTOW (fire control technician of the watch) rushed to complete his analysis of three surface contacts prior to PD, overlooking an updated 4000 yard closing solution on one of the 'old' contacts (the ill- 
fated Ehime Maru). His focus was entirely on a 'new' contact which he considered to be the primary contact of interest. The FTOW's lens was skewed by the false sense of urgency established by the $\mathrm{CO}$ which could very well have narrowed the FTOW's focus of attention. Further, when the FTOW heard the CO say he had a good feel for the contact picture, he assumed the $\mathrm{CO}$ was referring to all contacts, including the new one. This provides further evidence of a skewed lens on the part of the FTOW, which may have contributed to his decision to remain silent, failing to provide the $\mathrm{CO}$ with corrective information.

The CO's erroneous perception led to an incorrect comprehension (Oval 5) of the situation and an inaccurate projection (Oval 6) of where the vessels would be in the future. At no time did the $\mathrm{CO}$ discuss the surface picture with the contact management team to verify a common understanding of the surface contacts (Ovals 5 and 6, comprehension and projection). His own SA of contacts was based on two brief walk-throughs of the sonar room and a single review of fire control displays. He was overly confident, pressed for time, and failed to properly use both the technological agents and the human agents in the system to build his understanding of the surface picture.

The decision to proceed to PD represents a feedback loop from Oval 6 (projection) to Oval 1 (environment). As the submarine ascended to PD, the contents of Oval 1 were changed and new data were available for propagation through the model.

While at PD, the CO decided to interrupt the OOD's periscope search and performed his own abbreviated visual search for surface contacts. After the periscope searches by the OOD and CO, the FTOW cycled back through his surface contacts and correctly calculated a dangerous closing solution for one of the contacts, the Ehime Maru. However, the OOD had just stated he had seen no close contacts at $\mathrm{PD}$, and the $\mathrm{CO}$ also said he had no visual contacts. These pronouncements so skewed the lens of the FTOW that he doubted his comprehension (Oval 5) of the situation (that there was a surface contact in close proximity to the submarine).

\section{Actions of the FTOW}

The FTOW's erroneous comprehension generated a decision and a feedback loop from Oval 5 (comprehension) to Oval 3 (C2 workstation). He manually overrode the correct solution presented by his workstation, electronically changing the distance of the surface contact (Ehime Maru) from 4000 to 9000 yards, reflecting the distance to the visual horizon. The FTOW entered this erroneous information into the fire control system. The result of this action created an impossible speed solution of 99 knots. After the periscope searches, the boat went “emergency deep," proceeded to 400 feet, and conducted an emergency main ballast tank blow. The ship surfaced underneath the Ehime Maru, causing major flooding on that ship which sunk rapidly.

\section{CONCLUSION}

We have described various models of human error and proposed the Dynamic Model of Situated Cognition as another way to represent how decisions and events can propagate through a system. We believe the DMSC shows promise because it not only illustrates the linkages between technology and human agents but does so at a level and in a manner that gives investigators, researchers, and designers the ability to trace data through the system. We applied the DMSC by reviewing a recent submarine mishap involving the USS Greeneville. Analysis of this case revealed that the technological (e.g., sensor coverage and workstation display) and perceptual/cognitive (e.g., perception, comprehension, and projection) systems, along with the unique and evolving contents of the crewmembers' lenses, all played an important role in the unfolding of events. Error was injected at various critical stages and was propagated throughout the model to its ultimately disastrous ending.

This analysis, along with previous extensions of the model to the USS Stark (FFG 31) mishap (Shattuck \& Miller 2004), support the utility of an approach which integrates technological and human agents. This human - systems performance approach can inform system designers about weaknesses or vulnerabilities in a current or planned system and give them data they need to make modifications to the design, which, in turn will result in better systems and reduced risk to humans.

\section{REFERENCES}

Dekker, S. (2002). The field guide to human error investigations. Burlington, VT: Ashgate.

Dorner, D. (1989). The logic of failure: Recognizing and avoiding error in complex situations. Cambridge, MA: Perseus Books.

Executive Summary of USS Greenville collision, retrieved 04/14/2005 from http://www.cpf.navy.mil/greeneville html and http://www.cpf.navy.mil/pages/legal/foia/ Executive_Summary3.pdf.

Levinson, J. \& Edwards, R.L. (1997). Missile inbound: The attack on the Stark in the Persian Gulf. Annapolis, MD: Naval Institute Press.

Miller, N.L., \& Shattuck, L.G. (2004). A process model of situated cognition in military command and control. In Proceedings of the 2004 Command and Control Research and Technology Symposium, San Diego, CA.

Moray, N. (1994). Error reduction as a system problem. In M.S. Bogner (Ed.), Human error in medicine (pp. 67-91). Hillsdale, NJ: Lawrence Erlbaum Associates.

Moray, N. (2000). Culture, politics, and ergonomics. Ergonomics, 43, 858868.

Reason, J. (1990). Human error. Cambridge: University Press.

Reason, J. (1997). Managing the risks of organizational accidents. Burlington, VT: Ashgate.

Senders, J.W. \& Moray, N.P. (1991). Human error: Cause, prediction, and reduction. Hillsdale, NJ: Lawrence Erlbaum Associates.

Shattuck, L.G. \& Miller, N.L. (2004). A process tracing approach to the investigation of situated cognition. In Proceedings of the Human Factors and Ergonomics Society $48^{\text {th }}$ Annual Meeting (pp. 658-662). Santa Monica, CA: Human Factors and Ergonomics Society.

Snook, S. (2000). Friendly fire: The accidental shootdown of U.S. Blackhawks over northern Iraq. Princeton, NJ: Princeton University Press.

Strauch, B. (2002). Investigating human error: Incidents, accidents, and complex systems. Burlington, VT: Ashgate.

Wiegmann, D.A. \& Shappell, S.A. (2003). A Human Error Approach to Aviation Accident Analysis: The Human Factors Analysis and Classification System.Burlington, VT: Ashgate.

Woods, D.D., Johannesen, L.J., Cook, R.I., \& Sarter, N.B. (1994). Behind human error: Cognitive systems, computers, and hindsight. WrightPatterson Air Force Base, OH: Crew Systems Ergonomics Information Analysis Center. 